\title{
GPR Data Collection and Processing Strategies for Railway Ballast Evaluation
}

\author{
Luca Bianchini Ciampoli ${ }^{*}$, Alessandro Calvi ${ }^{*}$, Fabrizio D'Amico ${ }^{*}$ and Fabio Tosti ${ }^{\dagger}$ \\ "Department of Engineering, Roma Tre University, Rome, Italy \\ †School of Computing and Engineering, University of West London, London, United Kingdom \\ Email: luca.bianchiniciampoli@uniroma3.it
}

\begin{abstract}
Railways are important assets requiring continuous and effective monitoring. Within this context, nondestructive testing (NDT) methods are gaining momentum including, amongst others, the ground-penetrating radar (GPR) technique. GPR has proven its viability at providing effective condition-based assessment of railway ballast and identifying several different sources of decay. In this paper, the main challenges related to the data collection and processing stages for railway ballast investigations are reported. In addition, a review of main survey protocols and data processing strategies, including state-of-the-art research in this area of endeavor is presented, in terms of the issues related to the configuration of the track-bed structure (i.e., the effects of rails and sleepers on the signal) and the main inspection targets (i.e., ballast fouling, water content and segregation of the aggregates).
\end{abstract}

Keywords-GPR; railway monitoring; ballast decay; data processing; railway maintenance

\section{I.INTRODUCTION}

Monitoring of railway track-bed substructures are mostly carried out by traditional inspection methods, e.g., visual inspections or digging trenches at sections estimated as critical [1]. These methods are time-consuming and provide partial information on the condition of ballast [2]. Within this context, demand for more cost-effective and fast procedures in maintenance of railways has increased. Among the available non-destructive testing (NDT) methods used for the assessment of railway ballast, ground-penetrating radar (GPR) has emerged as one of the most popular techniques. The first attempt of GPR use in railway engineering dates back to 1985 $[3,4]$, and several applications have been reported over the last decades $[5,6]$.

\section{DATA COLLECTION AND PROCESSING ChALlENGES}

Acquisition, elaboration and interpretation of GPR data relative to the electromagnetic (EM) response of railway ballast is a matter of wide discussion within the research community. To this effect, railway structures are complex environments that may affect the collection of clear GPR dataset.

Amongst others, rebars in concrete sleepers heavily affect the informative content in a GPR signal as opposed to signals collected on ballast material only [7]. In case a GPR acquisition is performed on a typical railway track-bed, it is collected a sequence of ballast aggregates and concrete sleepers. To this effect, Fig. 1a represents typical time-domain-related effects exerted on the GPR signal by the presence of reinforced concrete sleepers. The source point for the emission of signals is here considered at the barycentre of the GPR apparatus (i.e., half-width of the GPR apparatus in the longitudinal crosssection scheme of Fig. 1a).
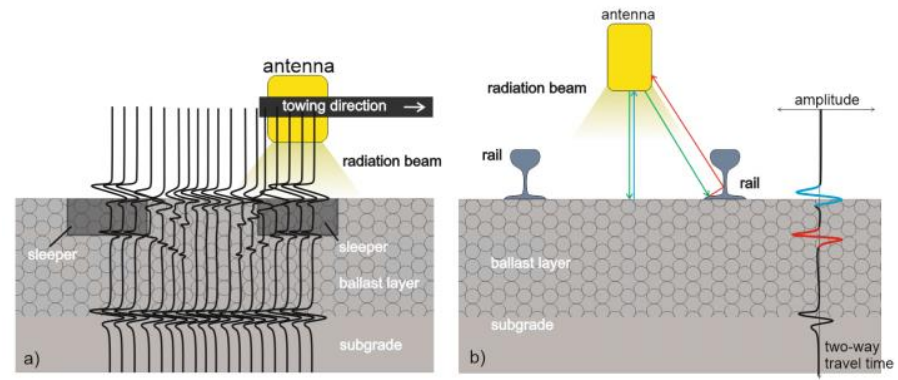

Fig. 1. a) Longitudinal cross-section: time-domain sequence of acquisition of GPR traces at the transition area between two consecutive concrete sleepers b) transversal cross-section: layout of the reflection patterns of a GPR signal collected in a ballast/rail system

Steel rails are another major source of clutter in GPR data. Fig. $1 \mathrm{~b}$ represents the time-domain effects on a transversal cross-section of the track. In case the steel element falls within the beam of radiation of the antenna, a further reflection peak (in red in Fig. 1b) will be observed in the A-scan trace. Since the rail is located farer from the receiver than the ballast surface directly below the antenna, the reception of this reflection will be slightly delayed with respect to the air-ballast surface reflection (in blue in Fig. 1b).

In addition to the above, other elements of complexity must be considered for interpretation purposes of a GPR signal in railway inspections. The dielectric properties of the layers composing the track-bed structure may be subject to large variability in short distances along the railway track. These variations are mainly related to the highly-variable conditions of the ballasted layers (due to local changes in fouling and water content conditions). The resulting changes in the values of dielectric permittivity and electrical conductivity can cause data interpretation errors or even inhibit penetration of the GPR signal towards the foundation layers.

Within this framework, the aforementioned effects must be adequately considered and, where possible, filtered out to achieve representative GPR data outputs. 


\section{SURVEY PROTOCOLS AND DATA PROCESSING STRATEGIES}

\section{A. Minimising noise from sleepers}

Sleeper-related effects on the signal are mainly governed by a change of reflectivity at the surface level (due to the material composition made by a randomly-distributed transition between aggregates and concrete) and the presence of a further reflection at the bottom of the sleeper. As the antenna moves away from a sleeper along the scanning direction, these effects progressively reduce and minimise at the barycentre of the sleeper/ballast transition area. As it is shown in Fig. 1, reflection effects from sleepers become therefore negligible at the mid-space between two consecutive sleepers, where reflections from the ballast material only are collected.

Although few research contributions exist about the minimisation of sleeper-related effects on the GPR signal by dedicated signal processing techniques [7], this is still an open issue.

To this effect, it is recommended to account for the influence of the antenna polarisation on the effects exerted by the sleepers on the signal.

In Fig. 2. it is shown a typical GPR signal collected over a railway track using a $1000 \mathrm{MHz}$ horn antenna and three different orientations, considered with respect to the longitudinal direction of the rails. The image represents a section with four concrete sleepers.
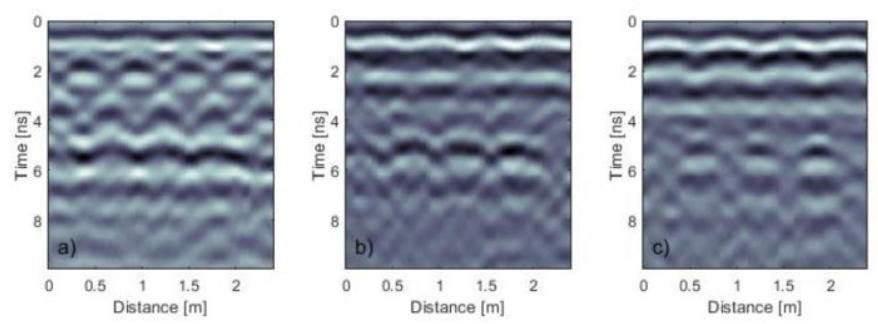

Fig. 2. Post-processed GPR radargams collected over a typical railway trackbed with a $1000 \mathrm{MHz}$ horn antenna with a) $0^{\circ}$, b) $45^{\circ}$ and c) $90^{\circ}$ orientations with respect to the longitudianldirection of the rails.

It is clearly visible that the $0^{\circ}$ orientation (Fig. 2a) returns the most affected radar image in terms of the effects exerted by the sleepers. This is due to the polarisation of the electromagnetic field being parallel to the orientation of the sleepers. Strong hyperbolic reflections can be also distinguished for each sleeper, at around $2 \mathrm{~ns}$.

On the opposite, , the $90^{\circ}$ orientation (Fig. 2c) provides the scan with less clutter, where the bottom of the ballasted layer between the sleepers $(\sim 5 n)$ is clearly visible. This is related to the particular orientation of the antenna, which makes it less affected by transversal objects.

The intermediate configuration (oblique polarisation, $45^{\circ}$ ) provided a radar image with an average degree of clutter, compared to the other two scenarios (Fig. 2b).

\section{B. Rail clutter filtering}

As it can be seen from the radargrams presented in Fig. 2, the distance between the antenna and the steel rails is a constant across the entire GPR survey. As a consequence, railrelated effects on the GPR signal can be represented by a constant reflection which is slightly delayed from that related to the air-ballast interface.

As the footprint of the antenna beam of radiation varies in the $x-y$ plan according to the antenna orientation, it is observed that orientation can also affect changes in the intensity of edge effects from targets placed at the radiation beam boundaries (e.g., the railway rails.).

Fig. 3 reports the average GPR traces referred to the sections presented in Fig. 2. It is relatively well-visible that for oblique $\left(45^{\circ}\right.$, orange line in Fig. 3$)$ and transversal $\left(90^{\circ}\right.$, yellow line in Fig. 3) orientations, reflections similar to ringing-noise effects occur after the first air-ballast reflection, i.e. in the range $\sim 7 \div 8 \mathrm{~ns}$, which are most likely caused by reflections from the rails. On the opposite, the longitudinal orientation $\left(0^{\circ}\right.$, blue line in Fig. 3) seems to be least affected by the effects of the rails.

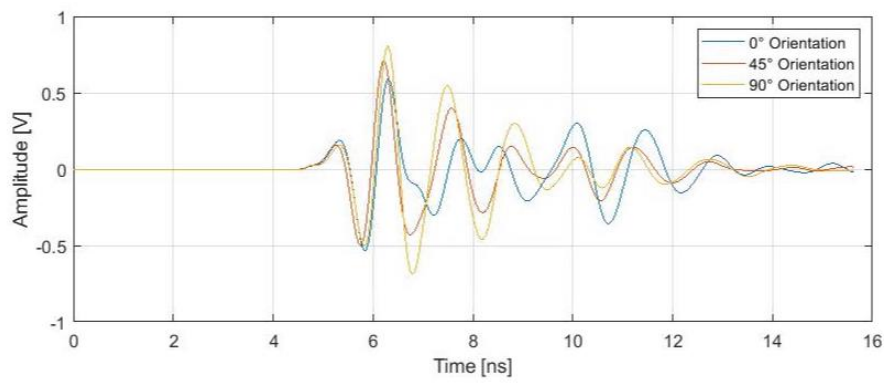

Fig. 3. Averaged post-processed GPR signals collected over a typical railway track-bed with a $1000 \mathrm{MHz}$ horn antenna with a) $0^{\circ}$, b) $45^{\circ}$ and c) $90^{\circ}$ orientations with respect to the longitudinal direction of the rails.

According to the time-constant character of the reflection from the rail, it is relatively easy to remove this clutter contribution from the raw signal. As it is shown in Fig. 4, the application of a background removal [8] (i.e. subtracting the average trace to the whole dataset along the $x$ direction) is generally sufficient to remove the effects of this factor.
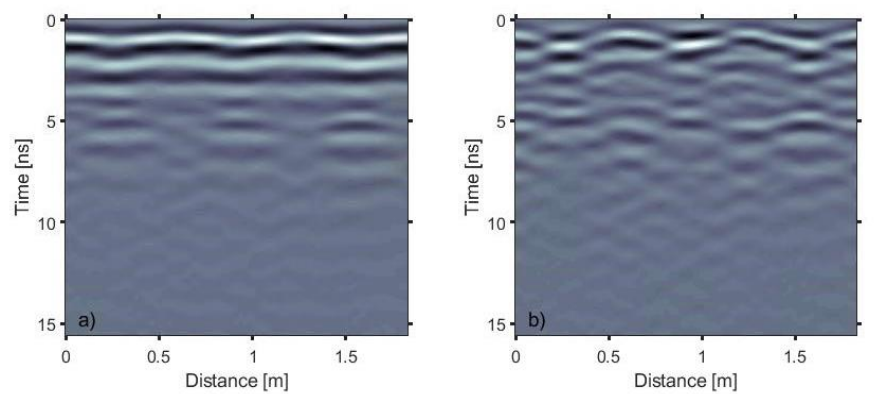

Fig. 4. Comparison of GPR scans collected with $90^{\circ}$ orientation (a) before and (b) after the application of the background removal. 


\section{Ballast fouling prediction}

Voids between ballast aggregates can get polluted by the deposit of several different materials, such as i) concrete and ii) steel dust produced at the ballast-sleeper and wheel-rail contact [9], respectively, iii) coal dust poured from the passing freights [10], and iv) fine clay particles raising from the subgrade along with capillary water [11]. Regardless of the nature of the process filling the ballast voids with fine particles, this occurrence is mostly referred to as 'fouling'.

Research on prediction of fouling in railway ballast has been widely reported in the past. Among the first, Hugenschmidt [12] performed a GPR survey over a railway section using a $900 \mathrm{MHz}$ antenna system. In this research, areas with an upward infiltration of clayey particles from the subgrade were detected. These were observed to affect strongly the depth of penetration of the GPR signal. The authors have claimed this occurrence to be relatively well recognisable, as the interface between clean and polluted ballast generates a visible reflection beyond which the signal gets quickly attenuated. A similar approach was followed in other research studies $[13,14]$.

Although the effects of clay on the attenuation of the EM waves are well-known [6], the occurrence of a clear reflection at the interface between the clean and polluted ballast is strictly dependent on the dielectric contrast between these materials. As an example, Fig. 5 shows a GPR survey conducted over a railway track in a section with a sharp variation of the ballast conditions. In more detail, the ballast is clean in the range $10 \div 16 \mathrm{~m}$, whereas voids are highly polluted by clay in the range $\div 20 \mathrm{~m}$. It is of evidence that no strong reflections are recognisable, whereas the presence of clay causes a visible attenuation of the signal.

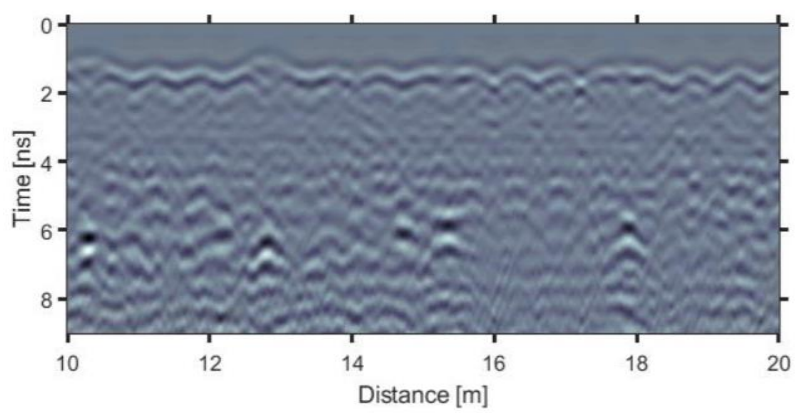

Fig. 5. Example of a $2000 \mathrm{MHz}$ GPR acquisition on a railway track with varying fouling conditions.

In view of the above, it is worth mentioning that the reliability of time-domain analyses is found to be highly dependent on the physical properties of the material composing the track-bed. As a consequence, more recent studies have been focusing on the influence of fouling on the EM response of the signal in the frequency domain.

Amongst the others, Methods for the detection of fouling on ballasted railway track-beds based on time-frequency approaches are presented in $[5,15,16]$. The time-dependent frequency properties can be inferred by decomposition of the signal using dedicated functions, in both time and frequency domains. To this extent, Al-Qadi et al. [15] applied both Discrete Wavelet Transform (DWT) and Short-Time Fourier Transform (STFT) to a GPR dataset collected over a real-life railway track. The authors successfully related the actual rate of fouling observed on site to the signal fluctuation level and the energy attenuation speed, respectively.

In view of the physio-chemical attitude of fine materials towards the retention of soil moisture [17], fouling and moisture are also expected to magnify each other's influence on the EM signal. In general, fouled ballast affects the efficiency of draining out liquids from the surface. In turn, the water retained by the fouling material heavily affects its dielectric properties. In this regard, the STFT algorithm was performed in [16] on GPR traces collected on a railway track-bed at varying fouling rates and water content levels, demonstrating the viability of the method in assessing the ballast fouling under different wet conditions.

An example of application of STFT on two railway tracks containing highly-fouled ballast at varying rates of moisture is reported in Fig. 6. In wet conditions (Fig. 6b), the higher frequency components are found to be almost totally attenuated within the first $10 \mathrm{~ns}$. Since the presence of fine particles attenuates the high-frequency portion of the EM signal, fouling conditions of ballast can be identified by observing the drop of energy at the high-frequency (from $1.5 \mathrm{GHz}$ to $5 \mathrm{GHz}$ in this case).
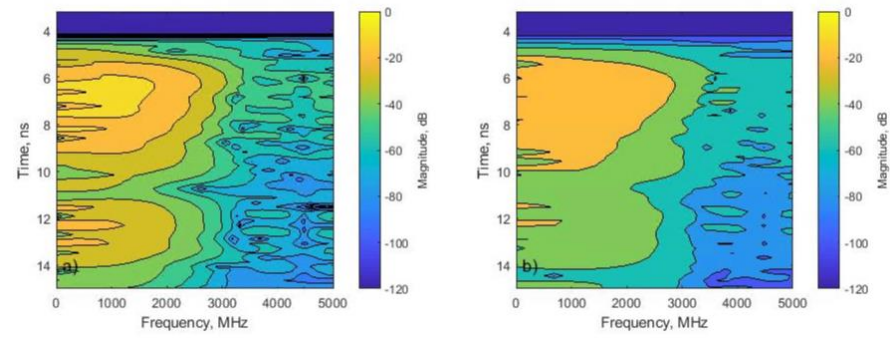

Fig. 6. Example of application of the STFT method on a $1000 \mathrm{MHz}$ GPR acquisition performed on a fouled railway track in a) dry and b) highly-wet conditions.

\section{Ballast fragmentation assessment}

Identifying change in the grading of railway ballast through EM methods is a challenging task, as no variations in the dielectric properties of the material are involved. Rock-derived aggregates composing the track-bed fragment over time due to compressive loads, and modify their size into smaller values. However, both sound and crushed aggregates are likely to fall beyond the resolution of the GPR systems usually employed for these purposes. This implies that visual observations of time-domain GPR signals can hardly identify segregation effects on ballast.

Few researches have focused on this particular topic. Liu et al. [18] have analysed the scattering attenuation loss and the velocity dispersion in the time domain of the signal with reference to the dimension of the granular aggregates. The authors compared the signal acquired over an experimental setup containing a sample of aggregates of known dimensions with the reference signal acquired on a metal plate. Even 
though the experiment succeeded in modelling the scattering loss of the signal propagating in the material, no quantitative relationship between this parameter and the material grading was proposed.

Similar to the research carried out for assessing fouling conditions, a frequency-based analysis of the problem has been performed in order to predict ballast fragmentation. Bianchini Ciampoli et al. [19] proposed an experimental-based model for predicting the dimension of the aggregates representing the whole track-bed grading. The model was based on the spectral analysis of the GPR traces. The authors stress out that the dimension of the ballast grains and the air-filled voids are related to scattering and resonance effects. These tend to amplify the natural frequency of the material and cause shifting of the peak of the spectrum.

Similar results have been reported more recently in [16]. On the base of an experimental activity carried out at the testsite scale level, authors confirm the relationship between the above frequency-based occurrences and high-frequency surveys (i.e. $2000 \mathrm{MHz}$ ). On the contrary, these were not detected using lower frequency antenna systems (i.e. 1000 $\mathrm{MHz}$ ).

\section{CONCLUSIONS}

The main challenges related to the data collection and processing stages for railway ballast investigations reported in this paper revealed that further research is still required to address more comprehensively the identification of main sources of decay. In particular, the review of the main survey protocols and data processing strategies has proven that several research efforts have been spent on removing the effects of rails and sleepers from the signal. Wavelength-resolution constraints related to the average size of ballast aggregates suggest that use of time-domain analyses of the GPR signal is not a viable approach in order to identify fouling or aggregatesegregation-related problems of the ballast material. On the contrary, use of methods based on the analysis of the spectral content of the GR signal seems to be a more robust approach in order to detect more comprehensive information in this area of endeavour.

\section{REFERENCES}

[1] Indraratna, B., 1st Proctor lecture of ISSMGE: railroad performance with special reference to ballast and substructure characteristics, Transportation Geotechnics, 7, 74-114, 2016.

[2] J. Hugenschmidt, "Railway track inspection using GPR," J. Appl. Geophys., vol. 43, no. 2, pp. 147-155, 2000.

The research is supported by the Italian Ministry of Education, University and Research under the National Project "Extended resilience analysis of transport networks (EXTRA TN): Towards a simultaneously space, aerial and ground sensed infrastructure for risks prevention", PRIN 2017, Prot. 20179BP4SM
[3] Roberts R, Schutz A, Al-Qadi IL, Tutumluer E. Characterizing railroad ballast using GPR: recent experiences in the United States. In Proceedings of the 2007 4th International Workshop on Advanced Ground Penetrating Radar (IWAGPR 2007), Naples, Italy, pp. 295-299, 2007.

[4] Railway Track and Structures Magazine, June 1985.

[5] Leng Z, Al-Qadi IL. Railroad ballast evaluation using groundpenetrating radar. Transp Res Rec 2010:2159;1110-17, 2010.

[6] A.Benedetto, F.Tosti, L. Bianchini Ciampoli, A. Calvi, M. G. Brancadoro, A. M. Alani , "Railway ballast condition assessment using ground-penetrating radar - An experimental, numerical simulation and modelling development”, Constr. Build. Mat.., vol. 140, pp. 508-520, Jun. 2017.

[7] L. Bianchini Ciampoli, S.S. Artagan, F. Tosti, A. Calvi, A.M. Alani, A. Benedetto. A GPR spectral-based filtering for minimisation of concrete sleepers effects in railway ballast investigations. Proc. of $41^{\text {st }}$ International Conference on Telecommunications and Signal Processing, 4-6 July, Athens, Greece, 2018

[8] L. Bianchini Ciampoli, F. Tosti, N. Economou, F. Benedetto, Signal Processing of GPR Data for Road Surveys, Geosciences (Switzerland), 9(96), 2019

[9] Indraratna, B., Nimbalkar, S., Coop, M. \& Sloan S.W. A Constitutive model for coal-fouled ballast capturing the ef-fects of particle degradation, In Computer and Geotechnics, 61, pp. 96-107, 2014

[10] Huang, H., Tutumluer, E., Hashash, Y., \& Ghaboussi, J. Laboratory validation of coal dust fouled ballast discrete el-ement model, In Paving Materials and Pavement Analysis, pp. 305-313, 2010.

[11] Al-Qadi, I.L., Xie, W., Roberts, R. \& Leng Z. Data analysis techniques for GPR used for assessing railroad ballast in high radio-frequency environment. In Journal of Trans-portation Engineering, 136(4), pp. 96105, 2010.

[12] J. Hugenschmidt, Railway track inspection using GPR, Journal of Applied Geophysics, 43, 147-155, 2000.

[13] Roberts, R., Al-Qadi, I.L., Tutumler, E., Kathage, A., Ballast fouling assessment u sing $2 \mathrm{ghz}$ horn antennas - gpr and ground truth comparis on from $238 \mathrm{~km}$ of track, Proc. of the 9th International Railway Engineering Conference, 2007

[14] Fontul, S., Fortunato, E., De Chiara, F., Evaluation of ballast fouling using GPR, Proc. of the 15 th international conference on ground penetrating radar (GPR 2014), 418-422, 2014

[15] I.L. Al-Qadi, S. Zhao, P. Shangguan, Railway ballast fouling detection using GPR data: introducing a combined time-frequency and discrete wavelet techniques, Near Surface Geophysics, 14 (2),145-153, 2016.

[16] Bianchini Ciampoli, L., Calvi, A., D’Amico, F., Railway Ballast Monitoring by GPR: A Test-Site Investigation, Remote Sensing, 11, 2381, 2019.

[17] Tosti F., Patriarca C., Slob E., Benedetto A. and Lambot S. Clay content evaluation in soils through GPR signal processing. Journal of Applied Geophysics 97, 69-80, 2013.

[18] Liu L, Li Z, Arcone S, Fu L, Huang Q. Radar wave scattering loss in a densely packed discrete random medium: numerical modeling of a boxof-boulders experiment in the Mie regime. Journal of Applied Geophysics,99:68-75, 2013.

[19] Bianchini Ciampoli, L., Tosti, F., Brancadoro, M.G., D'Amico, F., Alani, A.M., Benedetto, A., A spectral analysis of ground-penetrating radar data for the assessment of the railway ballast geometric properties, NDT\&E International, 90, 39-47, 2017 